\title{
Second Harmonlc Generation from Langmuir-Blodgett Films of Retinal and Retinal Schiff Bases
}

\author{
Jung Huang, Aaron Lewis, ${ }^{*}$ \\ Department of Applied Physics, Cornell University, Ithaca, New York 14853 \\ and Th. Rasing \\ Center for Advanced Materials, Lawrence Berkeley Laboratory, Berkeley, California 94720 \\ (Received: July 20, 1987)
}

\begin{abstract}
The second harmonic signal from monolayers of retinal and retinal Schiff bases is reported. The results have yielded information on the monolayer structure and demonstrate that retinal and retinal Schiff bases have large second-order molecular hyperpolarizabilities with values of $1.4 \times 10^{-28}, 1.2 \times 10^{-28}$, and $2.3 \times 10^{-28}$ esu for retinal, the unprotonated Schiff base, and the protonated Schiff base, respectively. These values compare well with the known variation in the alteration in the dipole moment of such chromophores upon excitation.
\end{abstract}

\section{Introduction}

The initial step in the process of visual excitation is the absorption of light by the chromophore of the visual pigment rhodopsin. In all visual pigments, the light absorbing entity is a derivative of retinal (vitamin A aldehyde). Thus, a detailed study of the excited-state properties of this retinylidene chromophore and related molecules is a crucial step in understanding the visual process.

Several spectroscopic techniques ${ }^{1}$ have been applied to investigate the photophysical and photochemical properties of the retinylidene chromophore. However, few nonlinear optical techniques have been used. Such methods can yield interesting information, as has been demonstrated in other systems. ${ }^{2,3}$ Second harmonic generation (SHG) is the lowest order nonlinear optical process, in which the second-order polarizability of a material is responsible for the generation of light at the second harmonic frequency. Due to symmetry consideration, the SHG is forbidden in an isotropic medium in the electric dipole approximation but allowed at the surface where the inversion symmetry is broken. It was recognized by Shen et al. ${ }^{4}$ that this attribute can be used to probe adsorbed monolayers at surfaces with high spatial and temporal resolution. Of great importance is the fact that this technique has been demonstrated to have submonolayer sensitivity, ${ }^{5,6}$ and recently Rasing et al. ${ }^{7}$ have proposed a simple and direct method to measure the second-order molecular polarizabilities of some organic molecules by using the surface second harmonic generation technique directly on a Langmuir-Blodgett trough.

Monolayers based on Langmuir-Blodgett troughs have attracted renewed interest during the last few years ${ }^{8}$ because such troughs allow the organization of appropriate molecules in a planned way so that the realization of artificial devices acting at the molecular scale becomes imaginable. The properties of biological membranes can readily be simulated by Langmuir-Blodgett (LB) monolayers. Second harmonic generation has the potential of being used as a direct, in situ surface probe of such monolayers. By use of this probe, detailed information can be obtained on the physical, chemical, and biological properties of these monolayers.

In this paper we apply SHG to compare the second harmonic properties of monolayers of retinal, retinylidene- $n$-butylamine Schiff base (NRB), and protonated NRB $\left(\mathrm{N}^{+} \mathrm{RB}-\mathrm{HCl}\right)$. The results have yielded new insight into the structure and dipolar properties of these molecules.

\section{Experimental Procedures}

A Q-switched frequency-doubled Nd:YAG laser with a $10-\mathrm{Hz}$ repetition rate and 10-ns pulse width was used. The Nd:YAG

* Address correspondence to this author at the Department of Applied Physics, Bergmann Building. The Hebrew University of Jerusalem, Jerusalem. Israel. laser was adjusted to give a green $532-\mathrm{nm}$ beam of less than 20 $\mathrm{mW}$ which was focused onto a 3-mm-diameter spot on the sample surface after passing through a half-wave plate, a Glan-Thompson laser prism polarizer, and a KG-5 color glass filter. The reflected second harmonic signal at a wavelength of $266 \mathrm{~nm}$ was passed through a UG-5 color glass filter which blocked the fundamental at $532 \mathrm{~nm}$. The UG-5 filter was followed by a UV Glan-Taylor polarizer and a Schoeffel $0.2-\mathrm{m}$ double monochromator. The SH signal was detected by a cooled RCA C 31034 photomultiplier and averaged by a boxcar integrator.

all-trans-Retinal purchased from Sigma was dissolved in $n$ hexane without further purification. High-pressure liquid chromatography indicated that the all-trans-retinal was greater than $95 \%$ pure. all-trans-Retinylidene- $n$-butylamine Schiff base (NRB) was prepared as described before. ${ }^{9}$ The molar concentrations of retinal and unprotonated and protonated NRB in methanol solutions were determined by the published extinction coefficients $\epsilon_{380}=4.2884 \times 10^{4} \mathrm{M}^{-1} \mathrm{~cm}^{-1},{ }^{10 \mathrm{a}} \epsilon_{365}=5.23 \times 10^{4} \mathrm{M}^{-1} \mathrm{~cm}^{-1}$, and $\epsilon_{445}=4.98 \times 10^{4} \mathrm{M}^{-1} \mathrm{~cm}^{-1},{ }^{10 \mathrm{~b}}$ respectively. The molecules were spread on the surface of deionized distilled pure water subphase at $\mathrm{pH} 6.4$.

The Langmuir trough was made out of Teflon. A movable barrier controlled the surface density of molecules, and no. 40 filter paper with dimensions of $0.7 \mathrm{~cm} \times 1.0 \mathrm{~cm}$ was attached to a Gould UC-3 force transducer to measure the surface pressure. During detection of the second harmonic signal, the movable barrier was controlled by a stepping motor such that the surface pressure of the film was kept at constant value.

Molecular orientation was determined by using the Heinz nulling technique. ${ }^{11}$ The incident pump beam was arranged to satisfy the condition

$$
\left[e_{\|}(\omega) / e_{\perp}(\omega)\right]^{2}=2
$$

(1) For a review see Methods in Enzymology; Packer, L. P., Ed.; Academic: New York, 1982; Vol. 88, Part I.

(2) Nestor, J. R. J. Raman Spectrosc. 1978, 7, 90

(3) Levenson, M. D. Introduction to Nonlinear Laser Spectroscopy; Academic: New York, 1982

(4) Shen, Y. R. Annu. Rev. Mater. Sci. 1986, 16, 69

(5) Tom, H. W. K.; Mate, C. M.; Zhu, X. D.; Crowell, J. E.; Heinz, T F. Phys. Rev. Lett. 1984, 52, 348

(6) Tom, H. W. K.; Mate, C. M.; Zhu, X. D.; Crowel1, J. E.; Shen, Y. R.; Somorjai, G. A. Surf. Sci. 1986, 172, 466

(7) Rasing, Th.; Berkovic, G.; Shen, Y. R.; Grubb, S. G.; Kim, M. W. Chem. Phys. Lett. 1986, 130, 1

(8) E.g., Second International Conference on Langmuir-Blodgett Films, Schenectady, New York, 1985; Zemel, J. N.; Ed.; In Thin Solid Films 1985, $132-134$.

(9) Marcus, M. A.; Lemley, A. T.; Lewis, A. J. Raman Spectrosc. 1979, 8,22 .

(10) (a) Becker, R. S.; Freedman, K. J. Am. Chem. Soc. 1985, 107, 1477

(b) Becker, R. S.; Freedman, K.; Lenoble, C. J. Phys. Chem. 1986, 90, 4334

(11) Heinz, T. F.; Tom, H. W. K.; Shen, Y. R. Phys. Rev. A 1983, 28 1983. 
<smiles>CC1=C(/C=C/C(C)=C/C=C/C(C)=C/C=O)C(C)(C)CCC1</smiles>

(a) All-trans Retinal

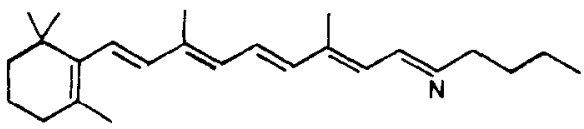

(b) All-trans Retinylidene n-Butylamine Schiff Base (NRB)<smiles></smiles>

(c) Protonated All-trans Retinylidene n-Butylamine Schiff Base (N $\mathbf{N}^{+} \mathrm{BB}-\mathrm{HCl}$ )

Figure 1. Chemical structures of (a) all-trans-retinal, (b) all-trans-retinylidene- $n$-butylamine Schiff base (NRB), and (c) protonated alltrans-retinylidene- $n$-butylamine Schiff base $\left(\mathrm{N}^{+} \mathrm{RB}-\mathrm{HCl}\right)$.

where $e(\omega)$ is the product of the polarization vector and the Fresnel factor for the pump field in the monolayer. ${ }^{12}$ The subscripts denote the components of $e(\omega)$ on or perpendicular to the layer plane. This condition can be met by adjusting the pump beam to have an angle of incidence of $45^{\circ}$ and a polarization of $35.3^{\circ}$ away from the incident plane. The molecular orientation was determined by the second harmonic extinction direction, as indicated by the UV polarizer.

Our detection sensitivity was calibrated against the second harmonic intensity from an $\mathrm{x}$-cut quartz plate, observed under the same experimental conditions. The quartz plate was rotated $28.75^{\circ}$ about the $y$ axis of the crystal to obtain one of the maxima of the Maker fringes. The polarizations of the fundamental and second harmonic beams were chosen to be p-polarized relative to the plane of incidence. In this case, the two beams are the extraordinary waves of the quartz crystal. Using the known nonlinear second-order susceptibility $d_{11}=2.4 \times 10^{-9} \mathrm{esu}^{13}$ and the index of refraction of quartz at 532 and $266 \mathrm{~nm}$, we obtained a ratio of ${ }^{14}$

$$
I_{\mathrm{p} \rightarrow \mathrm{p}}(2 \omega) /\left[I_{\mathrm{p}}(\omega)\right]^{2}=3.96 \times 10^{-26} \mathrm{~cm}^{2} \mathrm{erg}^{-1}
$$

The potential energy barrier between the water subphase and the NRB monolayer is quite small. This is particularly true for the NRB on an acid (pH 2.8) water subphase. We have observed that the unprotonated NRB molecules leak through the movable barrier on the acid water subphase during the compression stage. In addition, the proton on the Schiff base nitrogen of protonated NRB was found to be able to detach from the nitrogen. In order to avoid these problems, the second harmonic intensities from unprotonated and protonated NRB monolayers were compared by using spin-coated monolayers on glass substrates. ${ }^{15}$ The monolayers were carefully prepared to make sure that they have the same surface density of molecules. Several steps were taken to prevent the molecules on the glass substrates from isomerizing.

(12) Dick, B.; Gierulski, A.; Marowsky, G., Reider, G. A. Appl. Phys. B $1985,38,107$.

(13) Miller, R. C. Appl. Phys. Lett. 1964, 5, 17.

(14) (a) Jerphagnon, J.; Kurtz, S. K. J. Appl. Phys. 1970, 41, 1667. (b) Miller, R. C. Phys. Rev. 1963, 131, 95.

(15) Heinz, T. F.; Chen, C. K.; Ricard, D.; Shen, Y. R. Phys. Rev. Lett. 1982, 48,478 .

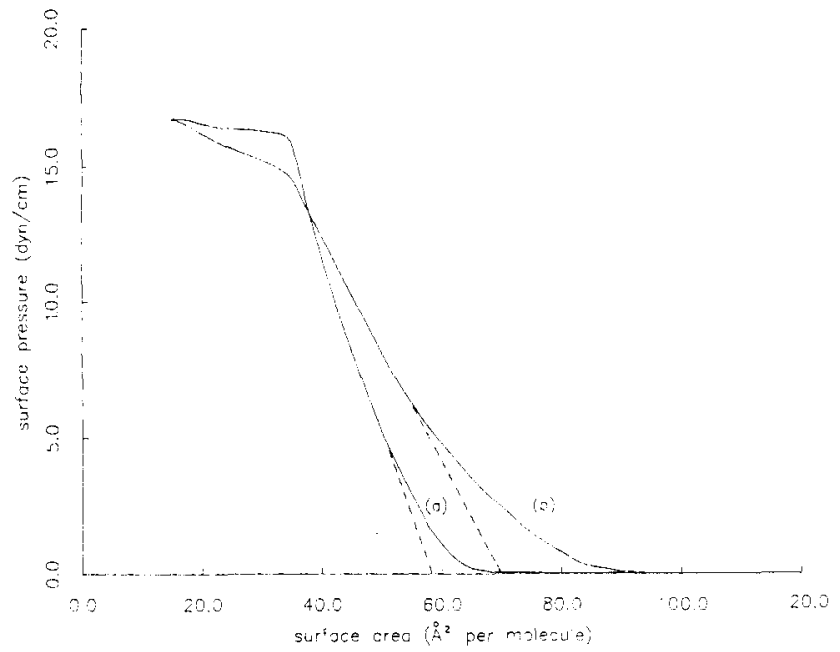

Figure 2. Surface pressure-surface area $(\pi-A)$ isotherms of (a) alltrans-retinal and (b) unprotonated retinylidene- $n$-butylamine Schiff base (NRB) Langmuir-Blodgett monolayers on a pure water subphase. The dashed lines indicate the extrapolations of the linear parts to zero pressure. The limiting areas are $58 \AA^{2}$ for retinal and $69 \AA^{2}$ for NRB. The cross point of these two isotherms is at the point where the surface pressure is $13 \mathrm{dyn} / \mathrm{cm}$ and the surface area is $38 \AA^{2} /$ molecule.

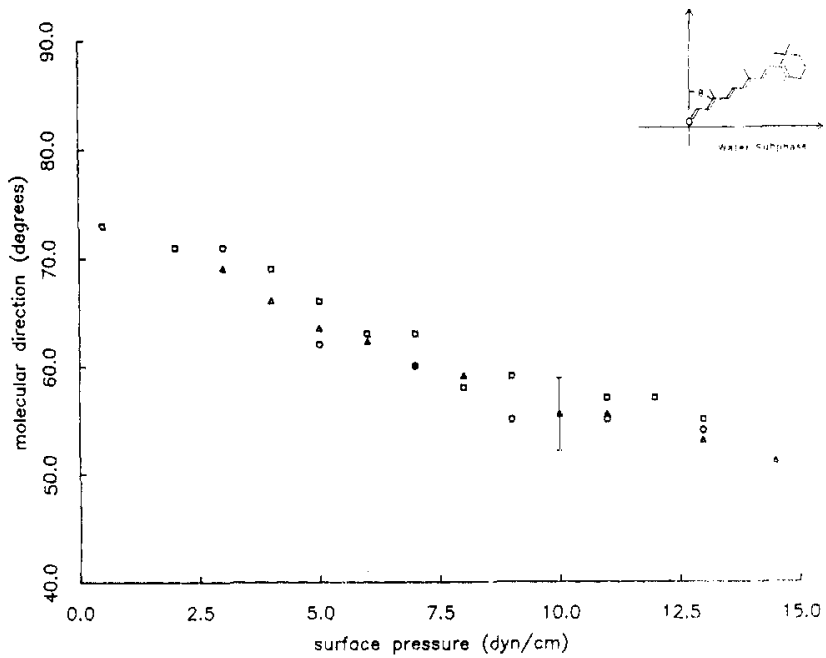

Figure 3. Molecular orientation at different surface pressures. The open circles and open boxes are for all-trans-retinal and retinal Schiff base (NRB) monolayers, respectively. The open triangles are for NRB on a $4 \mathrm{mM} \mathrm{CdCl}_{2}$ water subphase. The inset figure indicates the orientation parameter that was measured.

First, the average power of the pump laser was limited to below $15 \mathrm{~mW}$ when the spin-coated samples were measured. Second, the data collection time was reduced to $20 \mathrm{~s}$, and third, the samples were mounted on a spinning dc motor to minimize the exposure time of molecules to the pumping laser beam.

\section{Results and Discussion}

Correlation of Mechanical and Structural Properties of Retinal and Schiff Base Monolayers. The molecular structures of alltrans-retinal and its Schiff bases are shown in Figure 1. The initial characterization of the all-trans-retinal and unprotonated retinylidene- $n$-butylamine Schiff base (NRB) Langmuir-Blodgett films were made by measuring surface pressure-surface area $(\pi-A)$ isotherms. This allowed the mechanical properties of the films to be assessed. These measurements are shown in Figure 2 , where the curves $\mathrm{a}$ and $\mathrm{b}$ are for retinal and unprotonated NRB, respectively. The limiting areas $A_{\pi \rightarrow 0}$ obtained from extrapolation of the linear part to zero pressure ${ }^{16}$ were found to be $58 \AA^{2}$ for

(16) Nakahara, H.: Fukuda, K.; Möbius, D.; Kuhn, H. J. Phys. Chem. $1986,90.6144$ 


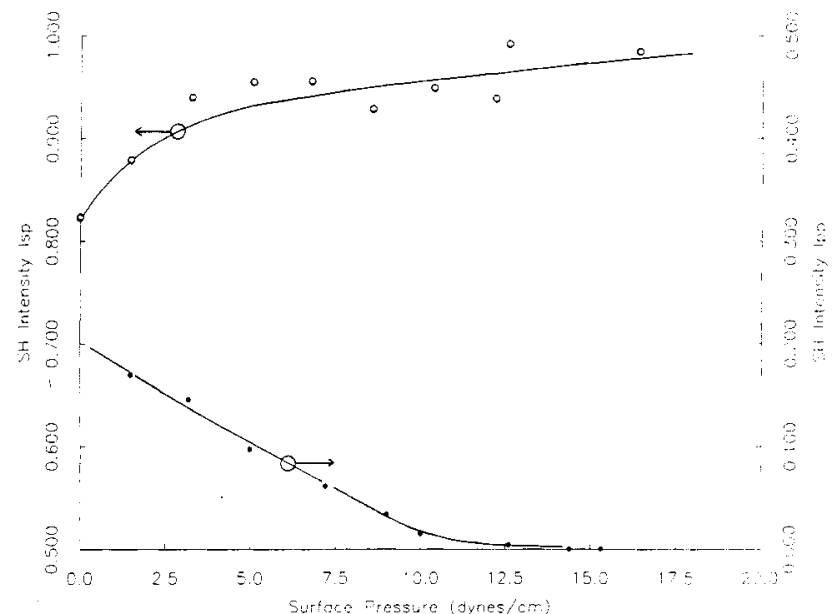

Figure 4. Second harmonic (SH) intensity of retinal monolayer at different surface pressures. The SH p-polarized intensity with an s-polarized pump beam, $I_{s \rightarrow p}$, which is proportional to the absolute square of the second-order susceptibility $\langle\beta\rangle_{2 x x}$ of the molecular monolayer whose surface coincides with the $x y$ plane, is shown in open circles. The solid circles are for $I_{\mathrm{p} \rightarrow \mathrm{p}} \mathrm{SH}$ intensity, which drops to zero when surface pressure is above $14 \mathrm{dyn} / \mathrm{cm}$. The solid lines through the points are only intended to guide the reader.

retinal and $69 \AA^{2}$ for unprotonated NRB. Based on the linear dimensions estimated from space-filling models of retinal, $18 \AA$, these limiting areas suggest the molecular long axes must tilt away from the water subphase surface. This observation is consistent with the results of the molecular orientation measurement, which are shown in Figure 3. The only difference between the chemical structures of retinal and unprotonated NRB is that the oxygen atom in retinal is replaced by a butylamine group (see Figure 1). The additional butyl group will require the NRB molecule to occupy a larger surface area than retinal does under the same surface pressure. However, this does not have any appreciable effect on the molecular orientation (see Figure 3 ). In this measurement the molecular directions of retinal and NRB are studied as a function of compressing the surface area and increasing the surface pressure. As is seen in this figure, for each of these molecules at low surface pressure a molecule direction of $\simeq 70^{\circ}$ relative to the surface normal is approached whereas at high surface pressure this angle reaches $\simeq 50^{\circ}$.

The cross section of the $\beta$-ionone ring on a plane perpendicular to the molecular long axis has dimensions of $5.2 \AA$ by $7.7 \AA$. If most of the steric interaction among molecules comes from the $\beta$-ionone ring, it would be expected that the LB films become closely packed at a surface area of $40 \AA^{2}$. Indeed, from Figure 2 , we observe that the isotherms of these two species level off below $32 \AA^{2}$. The slight difference of $8 \AA^{2}$ can be attributed to the dynamic property of the molecules on the water surface. In order to examine the structure of the film, we have measured the second harmonic intensity under different surface pressures. The results are shown in Figure 4 . It was observed that the SH p-polarized intensity $I_{\mathrm{s} \rightarrow \mathrm{p}}$ increases with the surface pressure in a nonlinear fashion. This is understandable by considering that the $\mathrm{SH}$ intensity is proportional to the absolute square of the second-order molecular hyperpolarizability $\beta$ by a coordinate transformation that depends on the molecular orientation: ${ }^{4}$

$$
\left(\chi^{(2)}\right)_{i j k}=N_{\mathrm{s}}\left\langle G_{l j k}^{\xi \xi}(\theta, \phi, \psi)\right\rangle \beta
$$

Here, $\langle G \eta \eta\rangle\rangle$ is the geometric factor specified by the coordinate transformation from the molecular coordinates $(\xi, \eta, \zeta)$ to the lab coordinates $(i, j, k)$, with the Euler angles $(\theta, \phi, \psi)$ describing the molecular orientation and the angular brackets indicating an average over all the molecules. From Figure 3, we found $\left\langle G_{i j k}^{\xi \eta \zeta}\right\rangle$ can depend on the surface pressure by way of the molecular orientation. Furthermore, the surface density $N_{\mathrm{s}}$ is not a linear function of the surface pressure as indicated in Figure 2. The SH intensity $I_{\mathrm{p} \rightarrow \mathrm{p}}$ on the other hand monotonically decreases with the surface pressure. It drops to zero at $14 \mathrm{dyn} / \mathrm{cm}$. The second

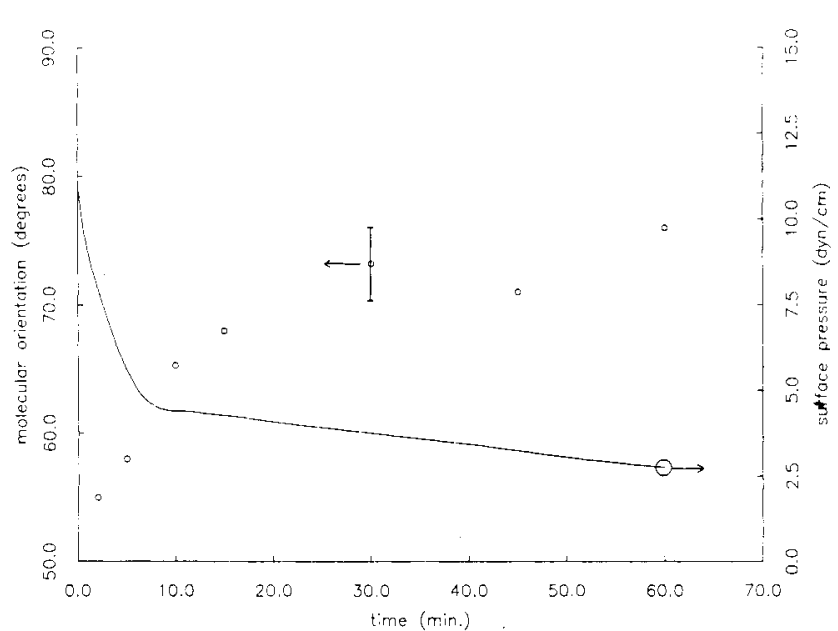

Figure 5. Relaxation behavior of the molecular orientation (open circles) and surface pressure (solid line) of the all-trans-retinal LB monolayer. The LB film was first compressed to $11 \mathrm{dyn} / \mathrm{cm}$ and then allowed to relax under the constant area condition.

harmonic p-polarized electric field strength $E_{\mathrm{p} \rightarrow \mathrm{p}}(2 \omega)$ is obtained in terms of the incident $\mathrm{p}$-polarized field: ${ }^{17}$

$$
\begin{aligned}
E_{\mathrm{p} \rightarrow \mathrm{p}}(2 \omega) \propto\langle\beta\rangle_{z x x}\left(\epsilon_{\mathrm{W}}-\sin ^{2} \alpha\right) & \left(1-\frac{2 \epsilon_{2}}{\epsilon_{1}}\right)+ \\
& \langle\beta\rangle_{z z z}\left(\sin ^{2} \alpha\right)\left(\frac{\epsilon_{\mathrm{W}}}{\epsilon_{1}}\right)^{2}
\end{aligned}
$$

where $\omega$ is the angular frequency of the incident radiation, $\alpha$ is the angle of incidence ( $45^{\circ}$ in our case), and $\epsilon_{W}, \epsilon_{1}$, and $\epsilon_{2}$ are the dielectric constants of the water subphase and of the film at $\omega$ and $2 \omega$, respectively. $\langle\beta\rangle_{z x x}$ and $\langle\beta\rangle_{z z z}$ are the two distinct nonvanishing average molecular hyperpolarizability components for the isotropic film considered here. For a rod-shaped molecule like retinal, the second-order molecular hyperpolarizability tensor is dominated by the one component corresponding to the incident and second harmonic electric fields lying along the axis of the molecule. If the molecular axes are inclined at an average angle $\theta$ to the subphase normal but have a random distribution of azimuthal angle, then

$$
\begin{gathered}
\langle\beta\rangle_{z z z}=\beta \cos ^{3} \theta \\
\langle\beta\rangle_{z x x}=\frac{1}{2} \beta \sin ^{2} \theta \cos \theta
\end{gathered}
$$

The vanishing value of $E_{\mathrm{p} \rightarrow \mathrm{p}}(2 \omega)$ implies that the inclination angle of the retinal molecule has a value of $59^{\circ}$. This is consistent with the molecular orientation measurement by the Heinz nulling technique (as described above and shown in Figure 3 ).

We have also observed the relaxation phenomenon of the all-trans-retinal LB film. The LB film was compressed to have $11 \mathrm{dyn} / \mathrm{cm}$ surface pressure and then was kept at the constant area condition. The time course of the molecular orientation and surface pressure were recorded during the film relaxation. We found that the molecular directions closely follow the surface pressure (see Figure 5). This suggests that the force that keeps the molecules standing up from the water surface mainly comes from the steric interaction between molecules and the same force takes part in generating the surface pressure.

Correlation of Second Harmonic Generation with Dipolar Properties of Retinal and Retinal Schiff Bases. Recent studies of nonlinear second-order optical processes in organic molecules have shown that two factors are important for a molecule to have large nonlinear optical response to an external applied field. ${ }^{18}$ The

(17) Girling, I. R.; Cada, N. A.; Kolinsky, P. V.; Montgomery, C. M. Electron Lett. 1985, 21, 169.

(18) Garito, A. F.; Singer, K. D.; Teng, C. C. In Nonlinear Optical Properties of Organic Materials; Williams, D. J., Ed.; American Chemical Society: Washington, DC, 1983. 
TABLE I: Comparisons of the Second-Order Molecular Hyperpolarizabilities and the Dipole Moment Changes of the First Excited $\pi \pi^{*}$ Singlet State for $a l l$-trans-Retinal and Unprotonated and Protonated Retinylidene- $n$-butylamine Schiff Bases

\begin{tabular}{|c|c|c|c|c|}
\hline \multirow[b]{2}{*}{ molecules } & \multirow{2}{*}{$\begin{array}{c}\chi_{z x x}^{(2)},{ }^{a} \text { esu } \\
\left(N_{\mathrm{s}}=2.5 \times 10^{14}\right)\end{array}$} & \multirow[b]{2}{*}{$\beta,^{b}$ esu } & \multicolumn{2}{|c|}{$\begin{array}{l}\text { excited-stated dipole } \\
\text { moment changes }{ }^{c}\end{array}$} \\
\hline & & & this work & ref 20 \\
\hline all-trans-retinal & $6.6 \times 10^{-15}$ & $(1.4 \pm 0.4) \times 10^{-28}$ & 1 & I \\
\hline unprotonated retinylidene- $n$-butylamine Schiff base (NRB) & $5.8 \times 10^{-15}$ & $1.2 \times 10^{-28}$ & 0.73 & 0.69 \\
\hline protonated retinylidene- $n$-butylamine Schiff base $\left(\mathrm{N}^{+} \mathrm{RB}-\mathrm{HCl}\right)$ & $1.1 \times 10^{-14}$ & $2.3 \times 10^{-28}$ & 0.97 & 1.03 \\
\hline
\end{tabular}

first is that molecules must have a highly polarizable $\pi$-electron system, and the second is that the $\pi$-electron system has to be distorted by interaction with strong electron donor and acceptor groups. Although retinal does not have explicit electron donor and acceptor groups, the charge transfer by way of the $\pi$-electron system can occur during the electronic excitation ${ }^{1} \mathrm{~B}_{\mathrm{u}} \leftarrow \mathrm{S}_{0}$. Quantum-chemical calculations ${ }^{19}$ have demonstrated that there is a dramatic change in charge distribution from the $\beta$-ionone ring to the carbonyl group such that the oxygen is more negative in the first excited singlet $\pi \pi^{*}$ state. Mathies et al. has used an electrochromic technique to monitor the electronic dipole moments of retinal and unprotonated and protonated NRB during the electronic excitation ${ }^{1} B_{u} \leftarrow S_{0} \cdot{ }^{20}$ Their data confirmed that retinal's condensed-phase dipole moment increases substantially $(14.7 \mathrm{D})$ upon excitation, with the unprotonated NRB having slightly less change (10.2 D) in dipole compared to retinal. In protonated NRB, the proton attached to the Schiff base nitrogen encourages the charge-transfer process and therefore increases the dipole moment change to $15.2 \mathrm{D}$. Charge transfer of this type should provide a substantial contribution to the second-order molecular hyperpolarizability. A simple two-level model has been used $^{21}$ to express this term as

$$
\beta=\frac{3 e^{2} \hbar^{2}}{2 m} \frac{W}{\left[W^{2}-(2 \hbar \omega)^{2}\right]\left[W^{2}-(\hbar \omega)^{2}\right]} f \Delta \mu_{\mathrm{ex}}
$$

where $\Delta \mu_{\mathrm{ex}}$ is the difference between excited- and ground-state dipole moments, $f$ is the oscillator strength of transition, $\hbar \omega$ is the fundamental photon energy, and $W$ is the energy of transition. If the charge-transfer process is the major mechanism in the second-order nonlinear optical response of retinal and unprotonated and protonated $\mathrm{NRB}$, the $\mathrm{SH}$ intensities from these three species should show the same increasing tendency from unprotonated NRB to retinal to protonated NRB as seen in the data of Mathies and Stryer. ${ }^{20}$ Table I summarizes our $\mathrm{SH}$ measurements for these molecules. We have found that the $\mathrm{SH}$ intensity from an unprotonated NRB LB film at a surface pressure of $13 \mathrm{dyn} / \mathrm{cm}$ is $70 \%$ of the $\mathrm{SH}$ intensity observed from a retinal LB film under the same conditions. By correcting for oscillator strength and energy dispersion factors, we have obtained a value of 0.73 for the ratio of the dipole moment changes between unprotonated NRB and retinal, close to the 0.69 value from the data of Mathies and Stryer. The SH signal from protonated NRB film is 3.7 times stronger than that of unprotonated NRB. After the same correction procedure, we have a ratio of 0.97 between protonated NRB and retinal whereas the Mathies and Stryer data gives a value of 1.03 . The consistency of our data with the work by Mathies and Stryer using the very different Stark shift technique is most encouraging.

By calibration the SH signal against a thin $\mathrm{x}$-cut quartz plate, the second-order molecular hyperpolarizability of retinal is es(19) Birge, R. R.; Hubbard, L. M. J. Am. Chem. Soc. 1980, 102, 2195,
(20) (a) Mathies, R.; Stryer, L. Proc. Natl. Acad. Sci. U.S.A. 1976, 73 , 2169. (b) Ponder, M.; Mathies, R. J. Phys. Chem, 1983, 87, 5090. (c) Davidsson, A.; Johansson, L. B. A. J. Phys. Chem. 1984, 88, 1094.

(21) Frazier, C. C.; Harrey, M. A.; Cockerham, M. P.; Hand, H. M.; Chauchard, E. A.; Lee, C. H. J. Phys. Chem. 1986, 90, 5703. Oudar, J. L.; Chemla, D. S. J. Chem. Phys. 1977, 66, 2664. timated to be $1.4 \times 10^{-28} \mathrm{esu}$, which is over 2 times larger than 2-methyl-4-nitroaniline $\left(\beta=4.5 \times 10^{-29} \mathrm{esu}\right.$ at $\left.0.83 \mu \mathrm{m}\right)$. Values of $1.2 \times 10^{-28}$ esu for unprotonated NRB and $2.3 \times 10^{-28}$ esu for protonated NRB were found. The large second-order molecular hyperpolarizability that we have measured and the near $55^{\circ}$ adsorbed direction ensure that such molecules are efficient $\mathrm{SH}$ generators. Recently Gierulski et al. ${ }^{22}$ has demonstrated a novel technique to measure the pulse width of a subpicosecond laser based on the $\mathrm{SH}$ generation from a dye-coated substrate. The time resolution is found to be limited by the transverse relaxation time $\left(T_{2}\right)$ of dye molecules. We have used a temporarily incoherent broad-band Stilbene-420 dye laser with 68 -fs correlation time to measure the incoherent photon echoes ${ }^{23}$ from protonated and unprotonated NRB. Our results show ${ }^{24}$ that the $T_{2}$ of protonated NRB at $420 \mathrm{~nm}$ is shorter than $15 \mathrm{fs}$. Our experiment suggests that retinal and protonated and unprotonated NRB can be very efficient time-correlation devices for subpicosecond light pulse measurements.

\section{Conclusion}

In conclusion, we have measured the second harmonic generation of retinal and unprotonated and protonated retinylidene$n$-butylamine Schiff bases at air-water and air-glass interfaces. Our data support the conclusions of other workers that the first excited singlet-state dipole moments of retinal and its Schiff bases have a major contribution from the charge-transfer process which takes place from the $\beta$-ionone ring to the end groups of these molecules. The long axes of the molecules tilt away from the surface normal by nearly $55^{\circ}$. The second-order molecular hyperpolarizability of protonated NRB is larger than unprotonated NRB, and the increment can be explained by the stabilization of the $\pi$-electron system provided by the proton which is attached to the Schiff base nitrogen. We believe that this technique could readily be extended to other relevant biological and chemical applications that require the investigation of dipolar and structural relationships between related systems.

The results reported in this paper are currently being extended to determine the light-induced alteration of the dipolar characteristic of the retinylidene chromophore in the membrane-bound bacteriorhodopsin. ${ }^{25}$

Acknowledgment. This work was supported partially by U.S. Army Contract No. DAMD17-85C-5136 and a grant from the U.S. Air Force (Grant AFOSR-87-0381) to A.L. and partially by the Director, Office of Energy Research, Office of Basic Energy Sciences, Materials Sciences Division of the U.S. Department of Energy under Contract No. DE-ACO3-76SF00098.

Registry No. NRB, 61769-47-9; N+RB-HCl, 61769-46-8; all-transretinal, 116-31-4.

(22) Gierulski, A.; Marowsky, G.; Nikolaus, B.; Vorobev, N. Appl Phys. $B$ 1985, 36, 133 .

(23) Nakatsuka, H.; Tomita, M.; Fujiwara, M.; Asaka, S. Opt. Commun. $1984,52,150$.

(24) Huang, J.; Lewis, A., unpublished results.

(25) Rasing, Th.; Huang, J.; Lewis, A.; Stehlin, T.; Shen, Y. R., to be published. 